\title{
PROOF OF THE NON-ISOMORPHISM OF TWO COLLINEATION GROUPS OF ORDER 5184*
}

BY F. A. LEWIS

Introduction. Let $S$ denote the collineation

$\rho x_{r}=\epsilon^{r-1} x_{r}^{\prime}, \quad(r=1, \cdots, n), \quad \epsilon=\cos (2 \pi / n)+i \sin (2 \pi / n)$,

and $T$ the collineation

$$
\rho x_{r}=x_{r+1}^{\prime}, \quad(r=1, \cdots, n), \quad x_{n+1}^{\prime} \equiv x_{1}^{\prime} .
$$

The abelian group $\{S, T\}$ of order $n^{2}$ is invariant under a group $\dagger$ $C_{n}$ of order

$$
n^{5}\left(1-\frac{1}{p_{1}^{2}}\right)\left(1-\frac{1}{p_{2}^{2}}\right) \cdots\left(1-\frac{1}{p_{m}^{2}}\right),
$$

where $p_{1}, p_{2}, \cdots, p_{m}$ are the distinct prime factors of $n$. The order of $C_{6}$ is 5184 .

Winger $\ddagger$ has discussed briefly the monomial group of order $(r+1) ! n^{r}$ that leaves invariant the variety

$$
x_{0}^{n}+x_{1}^{n}+x_{2}^{n}+\cdots+x_{r}^{n}=0 .
$$

This group is generated by the symmetric group of degree $r+1$ and an abelian group of order $n^{r}$ in canonical form. For $r=3$ and $n=6$ there results a group $G$ of order 5184 which has been treated by Musselman. $\$$ The purpose of this note is to prove that $G$ and $C_{6}$ are not simply isomorphic. The proof consists in showing that the number of collineations of period 2 in $G$ exceeds the number of collineations of period 2 in $C_{6}$.

* Presented to the Society, June 18, 1936.

$\dagger$ In fact, $C_{n}$ is the largest collineation group in $n$ variables containing $\{S, T\}$ invariantly, the coefficients and variables being in the field of complex numbers. (Author's dissertation, Ohio State University, 1934.)

$\ddagger$ Trinomial curves and monomial groups, American Journal of Mathematics, vol. 52 (1930), p. 394.

$\S$ On an imprimitive group of order 5184, American Journal of Mathematics, vol. 49 (1927). 
Proof of the Non-Isomorphism of $G$ and $C_{6}$. The group $C_{6}$ is generated by $\{S, T\}$ and the two collineations

$$
\begin{array}{ll}
V: & \rho x_{r}=\sum_{c=1}^{6} \epsilon^{(r-1)(c-1)} x_{c}^{\prime}, \quad(r=1, \cdots, 6), \\
W: & \rho x_{r}=\epsilon^{-(r-1)^{2} / 2} x_{r}^{\prime}, \quad(r=1, \cdots, 6),
\end{array}
$$

satisfying the following relations:

$$
\begin{array}{r}
V^{4}=W^{12}=1, V^{2} W=W V^{2}, V^{-1} S V=T^{-1}, W^{-1} S W=S, \\
(V W)^{3}=V^{2}=(W V)^{3}, W^{6}=S^{3}, V^{-1} T V=S, W^{-1} T W=S^{-1} T .
\end{array}
$$

The order of $H=\{V, W\}$ is 576. This group may be constructed by the following chain of invariant subgroups and an independent proof that the order of $C_{6}$ is 5184 follows readily.

$$
\begin{gathered}
H=\left\{V, G_{288}\right\}, \quad G_{288}=\left\{W^{5} V W^{3} V^{3}, G_{96}\right\}, \quad G_{96}=\left\{W^{2}, G_{32}\right\}, \\
G_{32}=\left\{W^{2}\left(W^{2} V\right)^{2}, G_{16}\right\}, \quad G_{16}=\left\{\left(W^{2} V\right)^{3} V, G_{4}\right\}, \quad G_{4}=\left\{S^{3}, T^{3}\right\} .
\end{gathered}
$$

Since $G_{4}$ is contained in $\{S, T\}$ which is invariant under $H$, the order of $C_{6}$ is $576 \cdot 36 / 4=5184$.

If $Q$, of order 144 , represents the quotient group of $C_{6}$ with respect to $\{S, T\}$, each element of $Q$, being a co-set of $C_{6}$, represents 36 collineations of $C_{6}$ that transform $\{S, T\}$ into itself according to the same isomorphism of $\{S, T\}$ with itself.* There are 24 collineations $S^{j} T^{k}$ of period 6 in $\{S, T\}$; if $S$ is transformed into a particular $S^{j} T^{k}$, the collineation $S^{l} T^{m}$ into which $T$ is to be transformed may be selected in six ways. Let $K$ represent a class of 144 collineations of $C_{6}$ corresponding to the 144 distinct possible sets $(j, k, l, m)$. That is, $K$ contains one and only one collineation from each of the 144 augmented co-sets of $C_{6}$. The square of $A \cdot S^{r} T^{s}$, an arbitrary collineation of the class $K$ from the co-set to which $A$ belongs, may be expressed in the form $A^{2} S^{u} T^{v}$ and hence is of period 2 only if $A^{2}$ is in $\{S, T\}$. That is, a necessary condition that $A \cdot S^{r} T^{s}$ be of period 2 is that $A^{2}$ be commutative with both $S$ and $T$. Among any class $K$ there are only 8 collineations $B$ such that the corresponding sets of values $(j, k, l, m)$ satisfy the congruences arising from the conditions that $B^{2}$ transform $S$ into $S$ and $T$ into $T$.

* It may easily be proved that the 36 collineations of $\{S, T\}$ are the only collineations in six variables commutative with both $S$ and $T$. 
The following table shows 8 such collineations, their squares, and the collineations of $\{S, T\}$ which multiply these 8 collineations on the right to form collineations of $C_{6}$ of period 2. The numbers in the last column show the total number of collineations of $C_{6}$ of period 2 corresponding to each $B$ of $K$. Thus it is seen that $C_{6}$ contains just 99 collineations of period 2 .

It is easily shown that $G$ contains more than 99 collineations of period 2 and hence $G$ and $C_{6}$ are not simply isomorphic.

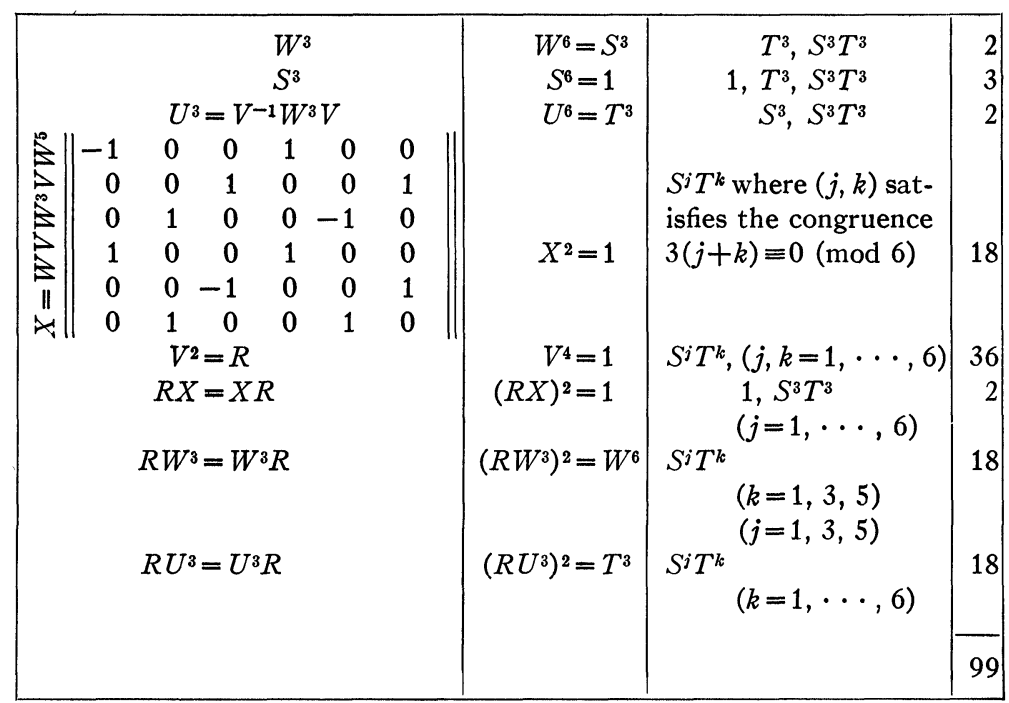

University of Alabama 